\title{
The role of cell signaling molecules in the pathogenesis of glomerulonephritis in children
}

\author{
${ }^{* 1,2}$ Angela Ciuntu \\ ${ }^{1}$ Department of Pediatrics, Nicolae Testemitanu State University of Medicine and Pharmacy \\ ${ }^{2}$ Institute of Mother and Child, Chisinau, the Republic of Moldova \\ Author's ORCID iD, academic degrees and contribution are available at the end of the article \\ *Corresponding author: angela.ciuntu@usmf.md \\ Manuscript received August 01, 2020; revised manuscript April 12, 2021; published online April 28, 2021
}

\begin{abstract}
Background: Cytokines are functional class of tiny proteins and glycoprotein and fundamentally they are monomers that function as soluble mediators in an autocrine or paracrine manner. Cytokines are produced by a number of cell types, predominantly leukocytes, and their targets implicate both immune and non-immune cells.

Material and methods: This study was performed on 75 children with glomerulonephritis (GN), aged from 2 up to 17 years. There were 20 children with steroid-sensitive nephrotic syndrome (SSNS), 15 children with steroid-resistant nephrotic syndrome (SRNS), 20 children with chronic glomerulonephritis (CGN) nephrotic form and 20 children with CGN mixed form. This study was performed on patients experiencing disease relapse and clinical remission. The control group consisted of 20 healthy children.

Results: The results of this study demonstrated increased levels of cell signaling molecules (IL-8, TNF- $\alpha$, MCP-1, MIP-1 $\alpha$ ) in the urine during clinical manifestations, valuable result due to their major role in the immunopathogenic mechanism of proteinuria in nephrotic syndrome.

Conclusions: Determination of urinary concentrations of cellular signaling molecules may be useful as a predictive non-invasive method for estimating disease activity, monitoring disease progression, differentiating steroid-sensitive nephrotic syndrome from steroid-resistant nephrotic syndrome, and assessing the effectiveness of treatment in children with different variants of GN.

Key words: cytokine, chemokine, nephrotic syndrome, glomerulonephritis.
\end{abstract}

Cite this article

Ciuntu A. The role of cell signaling molecules in the pathogenesis of glomerulonephritis in children. Mold Med J. 2021;64(2):37-41. https://doi.org/10.52418/ moldovan-med-j.64-2.21.07.

\section{Introduction}

Nephrotic syndrome (NS) is one of the most commonly encountered evidence of glomerular damage in children. It is characterized by a prevalence of approximately 16 cases per 100.000 children aged under 16 [1]. The reported annual incidence in children varies between 1.2 and 3.5 per 100.000 per year in Western Europe [2-4], 4.7 per 100.000 per year worldwide [5] and up to 6.5 per 100.000 per year in Japan [6]. NS is characterized by the triad of heavy proteinuria, hypoalbuminemia $(\leq 2.5 \mathrm{~g} / \mathrm{dL})$, and generalized edema. The pathophysiological mechanisms of idiopathic nephrotic syndrome (INS) support an underlying role for the immune system $[7,8]$. This hypothesis relies on the fact that the main treatment is based on corticosteroids and studies show different inflammatory profiles in patients with INS [9].

Idiopathic NS has been considered "a disorder of T-cell function" mediated by a circulating factor that alters podocyte function resulting in massive proteinuria for the last four decades.

The implicated role of circulatory factors released from T-cells has long been postulated in the pathophysiology of
NS; however, a single presumptive factor has not been defined yet [10].

Cytokines are functional class of tiny proteins and glycoprotein (molecular weights of approx. $8-80 \mathrm{kDa}$ ) and fundamentally they are monomers that function as soluble mediators in an autocrine or paracrine manner. Cytokines are produced by a number of cell types, predominantly leukocytes, and their targets implicate both immune and non-immune cells [11]. Also known as CXCL8, IL-8 is one of the most widely studied chemokines and is a critical inflammatory mediator. The main role of IL- 8 in inflammation is in the recruitment of neutrophils, although it is also responsible for the chemotactic migration and activation of monocytes, lymphocytes, basophils, and eosinophils at sites of inflammation [12].

Tumor necrosis factor (TNF), a pleiotrophic cytokine, that is produced primarily by immune cells, such as macrophages, dendritic cells, and $\mathrm{T}$ lymphocytes and is implicated in immune regulation [13]. TNF exerts its biological responses via interaction with two cell surface receptors: TNFR1 and TNFR2. (TNFRs) [14]. TNF is implicated 
in many systemic inflammatory diseases as well as kidney diseases [15]. TNF- $\alpha$ affects the expression of nephrin and podocyte cytoskeletal rearrangement [16].

Macrophage inflammatory protein (MIP)-1a/CCL3 is an inflammatory chemokine produced by cells during infection or inflammation. It belongs to the CC chemokine family, which displays potent chemotactic properties. Their findings suggested that the MIP-1 protein was composed of two peptides. Partial sequencing revealed two proteins: MIP-1 $\alpha$ and MIP-1 $\beta$. MIP-1 $\alpha / C C L 3$ is crucial for the recruitment of macrophages and T lymphocytes from the circulation to sites of infection or injury; thus, it orchestrates acute and chronic inflammatory host responses [17].

Monocyte chemoattractant protein-1 (CCL2/MCP-1) is a chemokine that mediates renal interstitial inflammation, tubular atrophy, and interstitial fibrosis by recruiting monocytes-macrophages into renal interstitium [18]. Within the glomeruli there is MCP-1 overexpression in both crescent GN and nephrotic conditions [19].

There are no good large-scale empirical evidence and complex studies regarding changes in cell signaling molecules in various clinically evolving variants of glomerulonephritis in children.

The aim of the study was to evaluate urinary concentration of cellular signaling molecules in children with glomerulonephritis with different clinical-evolutionary stages of the disease.

\section{Material and methods}

The prospective study was conducted in Nicolae Testemitanu State University of Medicine and Pharmacy, Department of Pediatrics, Biochemistry Laboratory and Institute of Mother and Child, Nephrology Unit.

It is based on biological samples collected according to the principles of contemporary research, approved by the Ethics Committee of Research of Nicolae Testemitanu State University of Medicine and Pharmacy (favorable review of 13.05.2015, official record No 55).

This study was performed on 75 children with glomerulonephritis, aged from 2 up to 17 years. There were 20 children with steroid-sensitive nephrotic syndrome (SSNS), 15 children with steroid-resistant nephrotic syndrome (SRNS), 20 children with chronic glomerulonephritis (CGN) nephrotic form and 20 children with CGN mixed form. At the first presentation, all patients received oral prednisone at dose of $2 \mathrm{mg} / \mathrm{kg} /$ day for 6-8 weeks. Diagnostic criteria for NS were based on the KDIGO Clinical Practice Guidelines for Glomerulonephritis [20]. This study was performed on patients experiencing disease relapse and during clinical remission. Inclusion criteria were: children aged 2-17 years, with primary nephrotic syndrome, CGN nephrotic and mixed form, endogenous creatinine clearance $>60 \mathrm{ml} / \mathrm{min}$ $/ 1.73 \mathrm{~m}^{2}$, and exclusion criteria: patients with congenital $\mathrm{NS}$, secondary NS, endogenous creatinine clearance $<60 \mathrm{ml}$ / min / $1.73 \mathrm{~m}^{2}$, cardiovascular, hepatic, metabolic comorbidities, acute injury of the internal organs at the time of study initiation. The control group consisted of 20 healthy, sex- and age-matched subjects.

Quantification of cytokines was performed by sandwich ELISA method. To assess the significant difference of performed indices were used statistical methods which appreciated arithmetic average size [X], average squared deviation, error of median arithmetic average size $[ \pm \mathrm{m}]$. It has also been used nonparametric statistical test "Mann-Whitney $U$ " and the selected level of statistical significance was $p$ $<0.05$ (StatsDirect statistical software, version 1.9.5, 2001).

\section{Results}

The mean value of age in NS (6.52 \pm 0.66 years old), CGN nephrotic form $(8.9 \pm 0.67$ years old) and CGN mixt form $(10.1 \pm 0.99$ years old). In the onset of the disease, clinical features were determined by anasarca - $(60.0 \pm 9.65 \%)$, edema - $(40.0 \pm 7.9 \%)$, rare urination $-(48.75 \pm 8.7 \%)$, headache - $(22.5 \pm 5.92 \%)$, vomiting - $(15.0 \pm 4.84 \%)$, abdominal pain syndrome $-(8.75 \pm 3.7 \%)$, pain in the lumbar region - $(10.0 \pm 3.95 \%)$. Evaluation of blood biochemical markers showed hypoproteinemia and hyperlipidemia in all subgroups of patients with NS (tab. 1).

Table 1. Laboratory findings of patients with idiopathic nephrotic syndrome according to the therapeutic response to steroids

\begin{tabular}{|l|c|c|}
\hline \multicolumn{1}{|c|}{ Biochemical parameters } & SSNS & SRNS \\
\hline Total protein, g/l & $53.3 \pm 1.27$ & $51.13 \pm 1.49$ \\
\hline Albumine, $\%$ & $35.72 \pm 3.56$ & $20.76 \pm 2.63$ \\
\hline Urea, mmol/l & $6.84 \pm 0.80$ & $5.12 \pm 0.48$ \\
\hline Creatinine, $\mathrm{mmol} / \mathrm{l}$ & $0.064 \pm 0.006$ & $0.054 \pm 0.037$ \\
\hline Total lipids, g/l & $9.20 \pm 1.13$ & $12.56 \pm 1.49$ \\
\hline Cholesterol, mmol/l & $7.43 \pm 0.34$ & $10.05 \pm 0.95$ \\
\hline$\beta$-lipoproteins, u.c. & $95.07 \pm 3.64$ & $107.23 \pm 6.88$ \\
\hline Protrombin, \% & $92.95 \pm 1.096$ & $92.50 \pm 1.71$ \\
\hline Fibrinogen, g/l & $4.59 \pm 0.38$ & $6.69 \pm 1.88$ \\
\hline $\begin{array}{c}\text { 24-Hour Urine Collection- pro- } \\
\text { teine, g/l }\end{array}$ & $3.79 \pm 0.66$ & $4.55 \pm 0.99$ \\
\hline
\end{tabular}

In table 2 are reflected results of the evaluation of urinary interleukins in patients with different forms of GN by estimating the level of IL-8 and TNF- $\alpha$.

Thus, in the onset period, in patients with SRNS we found an increased level of IL- 8 in urine that exceeded 9.5 times, and 4.7 times in SSNS compared to the control values. During remission, the level of IL- 8 in the urine remained increased in all clinical variants of $\mathrm{GN}$, compared to the control group.

Maintenance of high levels of IL-8 during remission in our research could be explained by decreased urinary clearance or slow degradation of this cytokine and persistence of the pathological process in the kidneys.

We obtained an increased TNF- $\alpha$ in the urine samples of all patients. The level of urinary TNF- $\alpha$ was more increased in the SRNS in the onset period, compared to the SSNS. 
Table 2. Urinary interleukin levels in children with glomerulonephritis ( $\mathrm{pg} / \mathrm{mM}$ creatinine)

\begin{tabular}{|c|c|c|c|c|}
\hline \multirow{2}{*}{ Study groups } & \multicolumn{2}{|c|}{ IL-8 } & \multicolumn{2}{|c|}{ TNF-a } \\
\hline & relapse & remission & relapse & remission \\
\hline SSNS & $\begin{array}{c}130.7 \pm 14.28^{* * * *} \\
465.1 \%\end{array}$ & $\begin{array}{c}86.0 \pm 8.94^{* * *} 306.04 \% \\
\mathrm{p}_{1}<0.05\end{array}$ & $\begin{array}{c}95.8 \pm 9.85^{* * *} \\
287.7 \%\end{array}$ & $\begin{array}{c}59.9 \pm 7.37^{* *} \\
179.9 \%, \mathrm{p}_{1}<0.05\end{array}$ \\
\hline SRNS & $\begin{array}{c}267.3 \pm 21.87^{* * *} \\
951.2 \%, p_{2}<0.001\end{array}$ & - & $\begin{array}{c}135.2 \pm 7.83^{* * *} \\
406.0 \%, \mathrm{p}_{2}<0.01\end{array}$ & $\begin{array}{c}82.3 \pm 10.34 \\
247.1 \%, p_{1}<0.01\end{array}$ \\
\hline CGN nephrotic form & $\begin{array}{c}291.0 \pm 25.73^{* * *} \\
1035.6 \%\end{array}$ & $\begin{array}{c}102.8 \pm 5.47^{* * *} 365.8 \% \\
\mathrm{p}_{1}<0.001\end{array}$ & $\begin{array}{c}62.3 \pm 5.72^{* * *} \\
187.1 \%\end{array}$ & $\begin{array}{c}22.1 \pm 0.78 \\
66.4 \%, \mathrm{p}_{1}<0.001\end{array}$ \\
\hline Control group & \multicolumn{2}{|c|}{$28.1 \pm 3.71$} & \multicolumn{2}{|c|}{$33.3 \pm 2.91$} \\
\hline
\end{tabular}

Note: statistically significant difference compared to control group values: ${ }^{\star} \mathrm{P}<0.05 ;{ }^{* *} \mathrm{P}<0.01 ;{ }^{* *} \mathrm{P}<0.001$.

$\mathrm{p}_{1-}$ authenticity compared with the same index registered under relapse. $\mathrm{p}_{2_{-}}$authenticity in comparison between SSNS and SRNS.

Thus, the level of urinary TNF- $\alpha$ increased 4.1 times in the SRNS and 2.9 times in the SSNS, compared to the control group, and during the remission the level of TNF- $\alpha$ in the urine, although decreased slightly, remains at increased values in these patients groups.

In CGN nephrotic form, the relapse period, there were also increased values of TNF- $\alpha$ that exceeded almost 2 times the control level. During the remission period, this parameter decreases, being by $33.6 \%$ lower than the values of the control group, but without statistical relevance.

We purpose to elucidate the disturbances in the cellular signaling system, so we evaluated the dynamics of urinary chemokines in patients with GN at different clinicalevolutionary stages of the disease (Table 3). The obtained results indicate a statistically authentic increase in the level of urinary MCP-1 in all groups of patients, compared to the control group, the highest values being recorded in children with CGN nephrotic and mixed form (Table 3).

The concentration of urinary MCP-1 in the groups of patients with SSNS and SRNS, during the clinical manifestations and remission period exceeded $2.0-2.4$ times the values of the control group. It should be noted that the group of patients with SRNS showed higher mean values of MCP1 in the urine compared to those in the group with SSNS.
In the groups of patients with CGN nephrotic and mixed form during exacerbation, the concentration of MCP-1 in the urine increased 3.3 times and 8.5 times, respectively, compared to the control group. During remission in groups of patients with CGN, the concentration of urinary MCP1 remains increased, which indicates the persistence of the chronic renal damage and the lack of a complete immunobiochemical recovery.

We found significantly higher levels of urinary MIP-1a in all groups of patients during the period of relapse compared to the period of remission. The level of urinary MIP$1 \alpha$ increased 2.7 times in the group of patients with SRNS, compared to SSNS during the clinical manifestations. In the group of patients with CGN nephrotic form, in the relapse period, the concentration of MIP-1 $\alpha$ in urine exceeded 4.9 times the level of this index recorded in patients with SSNS and 1.8 times in patients with SRNS.

\section{Discussion}

Our study revealed the clinical-diagnostic importance of assessing the concentration of cell signaling molecules in urine for the early diagnosis of renal impairment in children with GN, noting a significant increase in the concentration in their urinary samples. Furthermore, we obtained signifi-

Table 3. Urine chemokine levels of MCP-1 and MIP-1a in children with glomerulonephritis

\begin{tabular}{|c|c|c|c|c|}
\hline \multirow{2}{*}{ Study groups } & \multicolumn{2}{|c|}{ MCP-1 (pg/mM creatinine) } & \multicolumn{2}{|c|}{ MIP-1a (ng/mM creatinine) } \\
\hline & Relapse & Remission & Relapse & Remission \\
\hline SSNS & $\begin{array}{c}96.99 \pm 7.63^{* * * *} \\
190.4 \%\end{array}$ & $\begin{array}{c}112.13 \pm 6.88^{* *} \\
220.2 \%\end{array}$ & $\begin{array}{l}0.48 \pm 0.08 \\
p_{3}<0.001\end{array}$ & $\begin{array}{c}0.16 \pm 0.017 \\
p_{1}<0.05\end{array}$ \\
\hline SRNS & $\begin{array}{c}108.02 \pm 8.78^{* *} \\
212.1 \%\end{array}$ & $\begin{array}{c}121.88 \pm 7.26^{* * *} \\
239.3 \%\end{array}$ & $\begin{array}{c}1.28 \pm 0.09 \\
\mathrm{p}_{2}<0.01 \\
\mathrm{p}_{3}<0.01\end{array}$ & $\begin{array}{c}0.30 \pm 0.080 \\
p_{1}<0.01\end{array}$ \\
\hline CGN nephrotic form & $\begin{array}{c}170.32 \pm 12.01^{* * *} \\
334.4 \%\end{array}$ & $\begin{array}{c}93.21 \pm 8.43^{* *} \\
183.01 \%, \mathrm{p}_{1}<0.01\end{array}$ & $2.36 \pm 0.21$ & $\begin{array}{c}0.50 \pm 0.059 \\
p_{1}<0.001\end{array}$ \\
\hline CGN mixt form & $\begin{array}{c}430.76 \pm 49.73 \\
845.8 \%\end{array}$ & $\begin{array}{c}122.49 \pm 15.97^{*} \\
240.5 \%, p_{1}<0.01\end{array}$ & $7.58 \pm 2.72$ & - \\
\hline Control group & \multicolumn{2}{|c|}{$50.93 \pm 3.79$} & \multicolumn{2}{|c|}{0.0} \\
\hline
\end{tabular}

Note: statistically significant difference in relation to the values of the control group: ${ }^{\star} \mathrm{p}<0.05 ;{ }^{* *} \mathrm{p}<0.01 ;{ }^{* *} \mathrm{p}<0.001 . \mathrm{p}_{1}-$ authenticity in comparison with the respective index registered in relapse; $\mathrm{p}_{2}-$ authenticity when comparing SSNS with SRNS; $\mathrm{p}_{3}-$ authenticity when comparing CGN nephrotic form with SSNS and SRNS. 
cantly increased levels of IL- 8 in the urine in all groups of patients during the relapse period, compared to the remission period.

Previous studies had reported the relation between CXCL8/IL8 and disease activity and linked high urinary CXCL8/IL8 levels to proteinurea [21].

According to the study [22] urinary CXCL8/IL8 levels were significantly higher in SRNS than SSNS patients during activity and remission with no significant difference on comparing minimal change disease (MCD) and focal segmental glomerulosclerosis (FSGS) patients.

According to Souto M. et al. study, they found similar results, increased levels of urinary IL8/CXCL8 in relapsed steroid resistant children when compared to steroid sensitive patients in remission, with a positive correlation with urinary protein levels. These findings suggest that the renal release of the chemokine IL8/CXCL8 might be associated with changes in glomerular permeability [23].

IL-8 was shown to induce changes in the permeability of the glomerular basement membrane (GBM) via decreasing the synthesis of heparan sulfate proteoglycans, which eventually induced proteinuria in rats [24]. The study of Al-Eisa AA et al. detected increased urinary levels of IL- $1 \beta$, IL- 6 and IL-8 in INS patients during relapse which disappeared, except IL-8, during remission of the disease. These findings support the assumption of the important role of these cytokines in the immune process during a relapse [25].

A pediatric study used the TNF pathway in the recurrence of focal segmental glomerulosclerosis (FSGS) and showed improvement in proteinuria after TNF antibodies therapy [26].

During the precocious phases of inflammation, TNF- $\alpha$ may play as a key medium to activate the downstream factors expression, including $\gamma$-IFN, IL-1, IL-2, IL-4 and IL-8 and thus start a cascade reaction, which then promotes the nephrotic syndrome return by affecting glucocorticoid response [27].

The study of Besbas N. et al. suggests that increased urinary excretion of MCP-1 in the patients with FSGS is most likely due to enhanced production of MCP-1 in kidney, presumably induced by excessive exposure to plasma proteins filtered from the damaged glomeruli. They also showed that urinary MCP-1 levels correlated with the degree of proteinuria in FSGS patients [18].

Vianna and co-workers found higher urinary levels of MCP-1/CCL2 in patients with chronic kidney disease (CKD) due to FSGS than in cases of congenital uropathies [28]. Additionally, patients with glomerular disease had higher MCP-1 as compared with non-glomerular disease patients [28]. More recently, Matsumoto Y. et al. showed that urinary level of MCP-1/CCL2 was significantly higher in steroidresistant INS than in steroid-sensitive patients, supporting the idea that urinary MCP-1/CCL2 might contribute to the recruitment of macrophages into glomeruli [29].

Alzawa T. et al. in their study revealed that urinary concentrations of MCP-1 is a significant positive correlation with the degree of occult blood in urine and a significant inverse correlation with the estimated glomerular filtration rate. Furthermore, the urinary CCL2/MCP-1 concentration was significantly correlated with histological chronicity indices in patients with lupus nephritis and $\operatorname{IgA}$ nephropathy, supporting the hypothesis that the measurement of this chemokine may be useful as a noninvasive method for predicting the disease activity of glomerulonephritis in children [30].

The study of Ikezumi Y. et al. reported that macrophages play an important role in the pathogenesis of SRNS, showing a significant increase in glomerular macrophage accumulation in biopsies from children with SRNS compared with those with SSNS. These results suggest that MCP-1 could be involved in the mechanism for steroid-resistance via recruitment of monocytes/macrophages into kidney tissue [31].

According to the study of Furuichi K. et al. urinary levels of MIP-1a / CCL3 in patients with crescentric glomerulonephritis correlate with the percentage of cell crescents and the number of CD68-positive infiltration cells and CCR1- and CCR5-positive cells in glomeruli. Increased expression of MIP-1 $\alpha$, MIP-1ß and MCP-1 in glomeruli with cellular and fibrocellular crescents suggests that these chemokines may be involved in crescent progression additionally with macrophage recruitment. MIP-1 1 can specifically promote and generate glomerular infiltration with macrophages in the acute process leading to the formation of glomerular crescents [32].

\section{Conclusions}

Quantifying of urinary concentrations of cellular signaling molecules may be useful as a predictive non-invasive method for estimating disease activity, monitoring disease progression, differentiating steroid-sensitive nephrotic syndrome from steroid- resistant nephrotic syndrome, and assessing the effectiveness of treatment in children with different variants of glomerulonephritis.

\section{References}

1. Noone DG, Iijima K, Parekh R. Idiopathic nephrotic syndrome in children. Lancet. 2018;392:61-74. doi: 10.1016/S0140-6736(18)30536-1.

2. Franke I, Aydin M, Kurylowicz L, Lopez CEL, Ganschow R, Lentze MJ, Born M. Clinical course \& management of childhood nephrotic syndrome in Germany: a large epidemiological ESPED study. BMC Nephrol. 2019;20(1):45. doi: 10.1186/s12882-019-1233-1.

3. Franke I, Aydin M, Llamas Lopez CE, Kurylowicz L, Ganschow R, Lentze M, Born M. The incidence of the nephrotic syndrome in childhood in Germany. Clin Exp Nephrol. 2018 Feb;22(1):126-132. doi: 10.1007/ s10157-017-1433-6.

4. Dossier C, Lapidus N, Bayer F, Sellier-Leclerc AL, Boyer O, de Pontual L, May A, Nathanson S, Orzechowski C, Simon T, Carrat F, Deschenes G. Epidemiology of idiopathic nephrotic syndrome in children: endemic or epidemic? Pediatr Nephrol. 2016;31(12):2299-2308. doi: 10.1007/ s00467-016-3509-z.

5. Nandlal L, Naicker T, Bhimma R. Nephrotic syndrome in South African children: changing perspectives in the new millennium. Kidney Int Rep. 2019 Feb;12;4(4):522-534. doi: 10.1016/j.ekir.2019.01.019.

6. Kikunaga K, Ishikura K, Terano C, Sato M, Komaki F, Hamasaki Y, Sasaki S, Iijima K, Yoshikawa N, Nakanishi K, Nakazato H, Matsuyama T, Ando T, Ito S, Honda M. Japanese Pediatric Survey Holding Information of 
Nephrotic syndrome (JP-SHINE) study of the Japanese Study Group of Renal Disease in Children. High incidence of idiopathic nephrotic syndrome in East Asian children: a nationwide survey in Japan (JPSHINE study). Clin Exp Nephrol. 2016;21(4):651-657.doi: 10.1007/ s10157-016-1319-z.

7. Mallory LD, Claire G, Rulan SP, Damien GN. Nephrotic syndrome in infants and children: pathophysiology and management. Paediatr Int Child Health. 2017;37:248-258. doi: 10.1080/20469047.2017.1374003.

8. Pereira WF, Brito-Melo GE, Carneiro CM, Melo DS, Guimara es FL, Rocha-Vieira E, et al. Increased migratory and activation cell markers of peripheral blood lymphocytes in an experimental model of nephrotic syndrome. Mediators Inflamm. 2015;2015:209764. doi: $10.1155 / 2015 / 209764$.

9. Guimara es FTL, Melo GEBA, Cordeiro TM, Feracin V, Vieira ER, Pereira WF, et al. T-lymphocyte-expressing inflammatory cytokines underlie persistence of proteinuria in children with idiopathic nephrotic syndrome. J Pediatr. 2018;94(5):546-553. doi: 10.1016/j.jped.2017.08.005.

10. Yang Eun Mi. Pathogenesis of minimal change nephrotic syndrome: a review of the underlying molecular mechanisms. Child Kidney Dis. 2019;23(1):1-6. doi: 10.3339/jkspn.2019.23.1.1.

11. Sivangala R, Sumanlatha G. Cytokines that mediate and regulate immune responses. In: Khan WA. Innovative immunology. Irving: Austin publishing group; 2015. p. 1-26.

12. Turner MD, Nedjai B, Hursta T, Pennington DJ. Cytokines and chemokines: At the crossroads of cell signalling and inflammatory disease. Biochim Biophys Acta. 2014 Nov;1843(11):2563-2582. doi: 10.1016/j. bbamcr.2014.05.014

13. Pereira Wde F, Brito-Melo GE, Guimarães FT, Carvalho TG, Mateo EC, Simões e Silva AC. The role of the immune system in idiopathic nephrotic syndrome: a review of clinical and experimental studies. Inflamm Res. 2014;63(1):1-12. doi: 10.1007/s00011-013-0672-6.

14. Al-Lamki RS, Mayadas TN. TNF receptors: signaling pathways and contribution to renal dysfunction. Kidney Int. 2015;87(2):281-296. doi:10.1038/ki.2014.285.

15. Ernandez T, Mayadas TN. Immunoregulatory role of TNF alpha in inflammatory kidney diseases. Kidney Int. 2009 Aug;76(3):262-276. doi: 10.1038/ki.2009.142.

16. Rong C, Wenyan L, Yuanyuan W, Jian G. Advances and researches in idiopathic nephrotic syndrome biomarkers. MOJ Women's Health. 2017;5(1):184-187. doi: 10.15406/mojwh.2017.05.00110.

17. Bhavsar I, Miller CS, Al-Sabbagh M. Macrophage Inflammatory Protein-1 Alpha (MIP-1 alpha)/CCL3: as a biomarker. General Methods in Biomarker Research and their Applications. 2015:223-249. doi: 10.1007/978-94-007-7696-8_27.

18. Besbas N, Kalyoncu M, Cil O, Ozgul RK, Bakkaloglu A, Ozaltin F. MCP1 $2518 \mathrm{~A} / \mathrm{G}$ polymorphism affects progression of childhood focal segmental glomerulosclerosis. Ren Fail. 2015;37(9):1435-9. doi: 10.3109/0886022X.2015.1074474.
19. Yadav A, Saini V, Arora S. MCP-1: chemoattractant with a role beyond immunity: a review. Clin Chim Acta. 2010 Nov 11;411(21-22):1570-9. doi: 10.1016/j.cca.2010.07.006

20. Kidney Disease Improving Global Outcomes (KDIGO). Clinical practice guideline for glomerulonephritis. Kidney Int. 2012;2:S139-274.

21. Vivarelli M, Massella L, Ruggiero B, Emma F. Minimal change disease. Clin J Am Soc Nephrol. 2017 Feb;12(2):332-345. doi.org/10.2215/ CJN.05000516.

22. Heba MA, Osama EB, Rasha Abd El Razek Khattab, et al. Urinary Interleukin-8 as a biomarker for steroid resistance in childhood onset nephrotic syndrome. J Egypt Soc Pediatr Nephrol Transpl. 2019;14(1):9095. doi: 10.21608/GEGET.2019.38030.

23. Souto MF, Teixeira AL, Russo RC, et al. Immune mediators in idiopathic nephrotic syndrome: evidence for a relation between interleukin 8 and proteinuria. Pediatr Res. 2008 Dec;64(6):637-42. doi: 10.1203/ PDR.0b013e318186ddb2.

24. Pan Q, Wu J, Tao J, et al. Role of basophils in the pathogenesis of minimal change nephrotic syndrome: a literature review. Exp Ther Med. 2014 Oct;8(4):1027-1031. doi: 10.3892/etm.2014.1901.

25. Al-Eisa AA, Al Rushood M, Al-Attiyah RJ. Urinary excretion of IL-1 $\beta$, IL- 6 and IL- 8 cytokines during relapse and remission of idiopathic nephrotic syndrome. J Inflamm Res. 2017 Jan 23;10:1-5. doi: 10.2147/ JIR.S124947.

26. Bitzan M, Babayeva S, Vasudevan A, Goodyer P, Torban E. TNFa pathway blockade ameliorates toxic effects of FSGS plasma on podocyte cytoskeleton and $\beta 3$ integrin activation. Pediatr Nephrol. 2012 Dec;27(12):221726. doi: 10.1007/s00467-012-2163-3

27. Liang Y, Chen Y, Chen Y, Gong Y. Role of the glucocorticoid receptor in the recurrence of primary nephrotic syndrome. Exp Ther Med. 2015 Oct;10(4):1556-1562. doi: 10.3892/etm.2015.2665.

28. Vianna HR, Soares CM, Silveira KD, et al. Cytokines in chronic kidney disease: potential link of MCP-1 and dyslipidemia in glomerular diseases. Pediatr Nephrol. 2013;28(3):463-469. doi: 10.1007/s00467-012-2363-X.

29. Matsumoto Y, Ikezumi Y, Kondo T, Nakajima Y, Yamamoto Y, Morooka $\mathrm{M}$, et al. Urinary monocyte chemotactic protein 1 as a predictive marker of steroid responsiveness in children with idiopathic nephrotic syndrome. Fujita Med J. 2018;4:17-22. doi: 10.20407/fmj.4.1_17.

30. Aizawa T, Imaizumi T, Tsuruga $K$, et al. Urinary fractalkine and monocyte chemoattractant protein-1 as possible predictors of disease activity of childhood glomerulonephritis. Tohoku J Exp Med. 2013 Dec;231(4):265 70. doi: 10.1620/tjem.231.265.

31. Ikezumi Y, Suzuki T, Yamada T, Hasegawa H, Nikolic-Paterson DJ Saitoh A. Development of steroid-resistant pediatric nephrotic syndrome following prolonged steroid therapy is associated with alternative macrophage activation. Am Soc Nephrol. 2012;23:537A.

32. Furuichi K, Wada T, Sakai N, Iwata Y, et al. Distinct expression of CCR1 and CCR5 in glomerular and interstitial lesions of human glomerular diseases. Am J Nephrol. 2000;20(4):291-299. doi: 10.1159/000013603.

\section{Author's ORCID iD and academic degree}

Angela Ciuntu, MD, PhD, Professor of Pediatrics - https://orcid.org/0000-0003-4249-3555

Author's contribution

AC conceptualized the idea, conducted literature review, wrote the manuscript, revised and finalized the text.

\section{Funding}

The study was supported by Nicolae Testemitanu State University of Medicine and Pharmacy and Institute of Mother and Child. The author is independent and takes responsibility for the integrity of the data and accuracy of the data analysis.

\section{Ethics approval and consent to participate}

The research was approved by the Research Ethics Committee of Nicolae Testemitanu State University of Medicine and Pharmacy (protocol No 55 of May 13, 2015).

\section{Conflict of Interests}

No competing interests were disclosed. 